A review of the experimental and modeling development of a water phase change heat exchanger for future exploration support vehicles.

Authors: T. Cognata, T. Leimkuehler, R. Balasubramaniam, V. Nayagam, M. Hasan, R. Stephan

Water affords manifold benefits for human space exploration. Its properties make it useful for the storage of thermal energy as a Phase Change Material (PCM) in thermal control systems, in radiation shielding against Solar Particle Events (SPE) for the protection of crew members, and it is indisputably necessary for human life support. This paper envisions a single application for water which addresses these benefits for future exploration support vehicles and it describes recent experimental and modeling work that has been performed in order to arrive at a description of the thermal behavior of such a system. Experimental units have been developed and tested which permit the evaluation of the many parameters of design for such a system with emphasis on the latent energy content, temperature rise, mass, and interstitial material geometry. The experimental results are used to develop a robust and well correlated model which is intended to guide future design efforts toward the multi-purposed water PCM heat exchanger envisioned.

Add'l author contact information:

Balasubramaniam Ramaswamy, ramaswamy.balasubramaniam-1@nasa.gov

Vedha Nayagam, vedha.nayagam-1@nasa.gov

Mohammad Hasan, mohammad.m.hasan@nasa.gov

Ryan Stephan, ryan.a.stephan@nasa.gov 


\title{
A Review of the Experimental and Modeling Development of a Water Phase Change Heat Exchanger for Future Exploration Support Vehicles
}

\author{
Thomas J. Cognata ${ }^{1}$ \\ MEI Technologies, Houston, TX, 77058 \\ Thomas Leimkuehler ${ }^{2}$ \\ Paragon SDC, Houston, TX, 77058
}

R. Balasubramaniam ${ }^{3}$, Vedha Nayagam ${ }^{4}$, and Mohammad Hasan ${ }^{5}$

NASA Glenn Research Center, Cleveland, OH, 44135

and

Ryan Stephan ${ }^{6}$

NASA Johnson Space Center, Houston, TX, 77058

\begin{abstract}
Water affords manifold benefits for human space exploration. Its properties make it useful for the storage of thermal energy as a Phase Change Material (PCM) in thermal control systems, in radiation shielding against Solar Particle Events (SPE) for the protection of crew members, and it is indisputably necessary for human life support. This paper envisions a single application for water which addresses these benefits for future exploration support vehicles and it describes recent experimental and modeling work that has been performed in order to arrive at a description of the thermal behavior of such a system. Experimental units have been developed and tested which permit the evaluation of the many parameters of design for such a system with emphasis on the latent energy content, temperature rise, mass, and interstitial material geometry. The experimental results are used to develop a robust and well correlated model which is intended to guide future design efforts toward the multi-purposed water PCM heat exchanger envisioned.
\end{abstract}

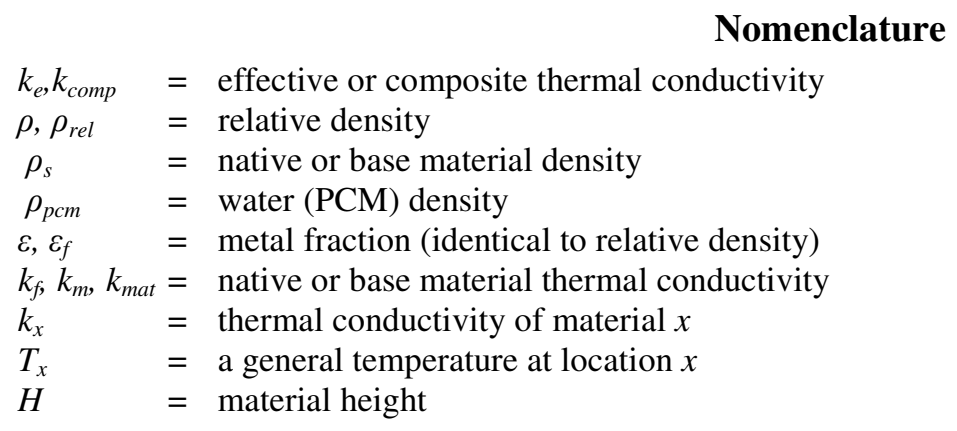

\footnotetext{
${ }^{1}$ Sr. Engineer, Engineering \& Science Contract Group, 2224 Bay Area Blvd.

${ }^{2}$ Sr. Aerospace Engineer, 1120 NASA Parkway, Suite505, AIAA Member.

${ }^{3}$ Research Associate Professor, Nat'l Center for Space Exploration Research, Case Western Reserve Univ./NASA GRC, M/S 110-3 21000 Brookpark Rd.

${ }^{4}$ Research Associate Professor, Nat'l Center for Space Exploration Research, Case Western Reserve Univ./NASA GRC, M/S 110-3 21000 Brookpark Rd.

${ }^{5}$ Aerospace Engineer, M/S 77-5, 21000 Brookpark Rd.

${ }^{6}$ Thermal Engineer, Crew and Thermal Systems Division, 2101 NASA Parkway, M/S EC2, AIAA Member.
} 


$\begin{array}{ll}C & =\text { a general constant } \\ C_{p x} & =\text { heat capacity of material } x \\ R_{m} & =\text { test article thermal resistance to ambient } \\ l & =\text { length of on side of a honeycomb cell } \\ L & =\text { characteristic length of a material } \\ c & =\text { cell size of honeycomb material } \\ r_{c} & =\text { honeycomb equivalent radius } \\ t_{c} & =\text { honeycomb equivalent wall thickness } \\ q, q " & =\text { heat flux } \\ q_{f} & =\text { heat flux through the fin } \\ Q_{\text {heater }} & =\text { heat applied by the heater } \\ Q_{\text {fluid }} & =\text { heat applied by the fluid loop } \\ T_{m} & =\text { the mean test article temperature } \\ t, t h & =\text { wall thickness, characteristic thickness of a material } \\ t_{\text {freeze }} & =\text { time to freeze } \\ t_{\text {melt }} & =\text { time to melt } \\ m & =\text { mass } \\ W & =\text { width of a unit cell } \\ \Delta t & =\text { time step } \\ \hat{L} & =\text { latent heat for phase change } \\ V_{\text {front }} & =\text { velocity of the phase change front during melt/thaw } \\ v_{f f} & =\text { velocity of the phase change front during solidification } \\ \Delta y & =\text { distance along y axis }\end{array}$

\section{Introduction}

$\mathrm{T}$ hermal control system (TCS) design for space exploration vehicles must account for its most extreme environment. Where heat is rejected by a radiator, for example, the radiator must be sized to reject in the warmest environment that the vehicle is expected to experience. For a planetary crew transport it would be designed to reject peak load in a subsolar point environment. When sized thus, a crew transport designed to support multiple crew-members and their equipment might be overwhelmed by the size and weight of a radiator which is overadaquate for most of its missions. The use of Phase Change Material (PCM) heat exchangers in a TCS has historically been used to mitigate such design issues by utilizing the latent heat of fusion of a material to temporarily store heat generated during peak use or while in hot environments in order to size a smaller radiator appropriate for an average mission heat load and environment.

In extreme environments that do not afford adaquate protection, such as in interplanetary travel, on Mars, the Moon, or on asteroids, it has been proposed that crewmembers might be protected from radiation such as due to Solar Particle Events (SPE) by an approximately 4-inch layer of water lining exterior walls. This proposed water layer can also be frozen by heat rejection from the exterior surface of the vehicle or via a radiator and used thus as a PCM for a multi-purposed heat exchanger integral to a vehicle. Such a volume of water can also afford emergency supplies of the vital fluid for crew survival or as an expendable in a topping heat rejection device such as in a sublimator or evaporator heat exchanger which are currently in use or in development for use in suit and vehicle TCS's.

Such a multi purposed heat exchanger might be designed such that the exterior of the vehicle behaves as the radiator to reject heat and the interior surface acts to exchange heat with the cabin air. The PCM layer would fill the gap between, rejecting heat to the radiator and storing excess heat in the phase change of the water from solid to liquid while the vehicle is on-mission. When the vehicle's mission is complete, it would be left in a cold environment to regenerate (freeze) the PCM for the vehicle's next mission. This project considers just such a design and the materials inherent in a well considered PCM, the interstitial materials which facilitate the transport of heat to and through the PCM in such a heat exchanger.

A previous investigation ${ }^{1,2}$ examined heat input and removal from different sides of the PCM. The current investigation takes a more thorough look at optimizing the interstitial material. A one-dimensional analysis ${ }^{3}$ is sufficient for providing an initial estimate for the amount of interstitial material to include with the PCM given a specific set of requirements. However, a more detailed analysis is necessary to evaluate the optimal material and the optimal geometry of the interstitial material. The current investigation aims to address these issues. 


\section{Experimental Setup}

The system and facilities described in this section are purpose designed for this experimental program. The experimental system is composed of a test article developed to accommodate many different interstitial material types, a chilled fluid loop which provides a low temperature sink, a heater and power supply which adds thermal energy to the system, and a data acquisition system for the recording of data and control of test conditions. The experiment is performed in the Advanced Thermal Control Systems (ATCS) lab in the NASA Johnson Space Center (JSC) Building 7 high-bay. The following sections describe the major components of the experimental setup in greater detail.

\section{A. Test Article Design}

This test is designed to accommodate the evaluation of many different interstitial material types, including folded fins, honeycomb, and foams, all having various parameters. The test is thus designed in a modular fashion such that each instrumented interstitial material may be dropped into identical test articles which apply heat flux, contain the phase change material, and insulate the test from ambient conditions. Each test article is an assembly, as shown in Figure 1 , which includes a container, a heater, the interstitial material, thermal interface materials, heat spreaders, and a cold plate.

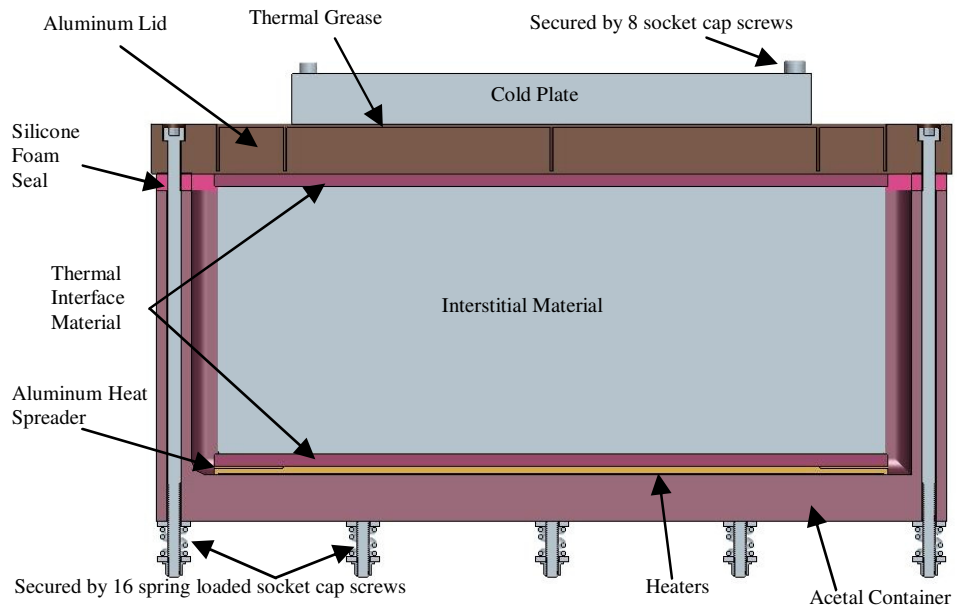

The cold plate connects to a

chilled fluid loop for the removal of heat energy from the system. The aluminum lid acts as a heat spreader to the coldplate to ensure a constant temperature upper bound to the interstitial material. At the interstitial material face opposite the cold plate is a heater and heat spreader assembly. A thermal interface material, a specially formulated silicone pad, is placed between the interstitial material and heat spreaders and the assembly is spring loaded to ensure consistent thermal contact for all test conditions. Test articles contain $\sim 80 \%$ deionized (DI) liquid water by volume at room temperature. As a result, test articles are estimated to contain, due to expansion upon phase change, approximately $90 \%$ DI solid water (ice) by volume at and below $0^{\circ} \mathrm{C}$. The test article is surrounded by an insulation package as illustrated in Figure 3.

\section{B. Test Article Instrumentation}

Each test article is instrumented with 28 and each insulation package with 2 type $\mathrm{T}$ thermocouples. Twenty-four of these are recorded at regular intervals via a National Instruments (NI) fieldpoint data acquisition system (DAQ). The instrumentation of the test article is shown in Figure 2.

The interstitial material is instrumented along four columns, three identified by lines in the figure, and the fourth offset from the center

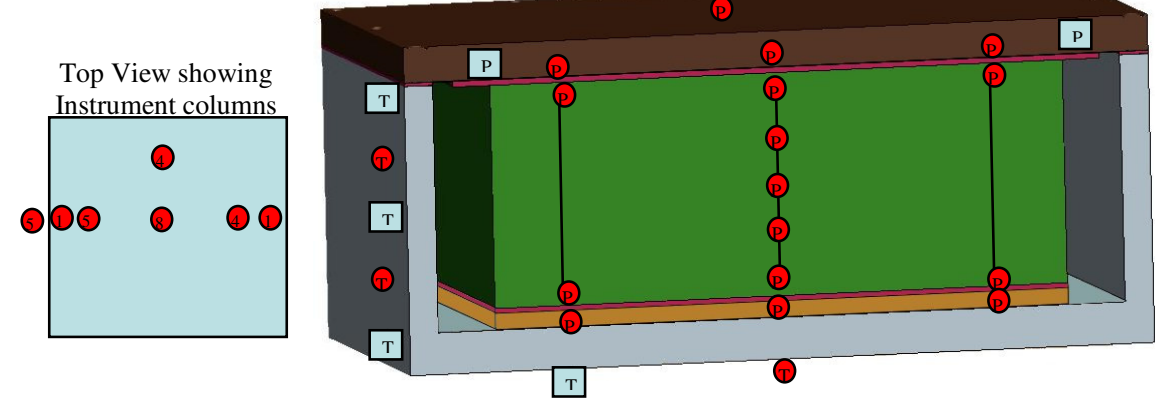

Figure 2 Test article instrumentation. $P$ indicates a probe, $T$ a beaded thermocouple. Only red locations are monitored by the DAQ. 
column perpendicular to the plane made from these three columns. The central column contains 5 thermocouple probes, one located at the top center (TC) of the material, a second at the bottom center (BC) of the material, and the remaining three spaced evenly, approximately 1" apart, between these two $(\mathrm{C} 1, \mathrm{C} 2$, and $\mathrm{C} 3)$. Each of the three other columns is spaced 4" distance from this central column with a single probe located at the top and a single probe located at the bottom of the material. The interstitial material accounts for 11 of the total thermocouple count.

The heater is instrumented with 4 type $T$ thermocouples, each of these, when the test article is assembled, at locations corresponding to the columns in the interstitial material. The lid is similarly instrumented, but with two additional type $\mathrm{T}$ thermocouples located 5 in distant from the center which are not recorded by the DAQ.

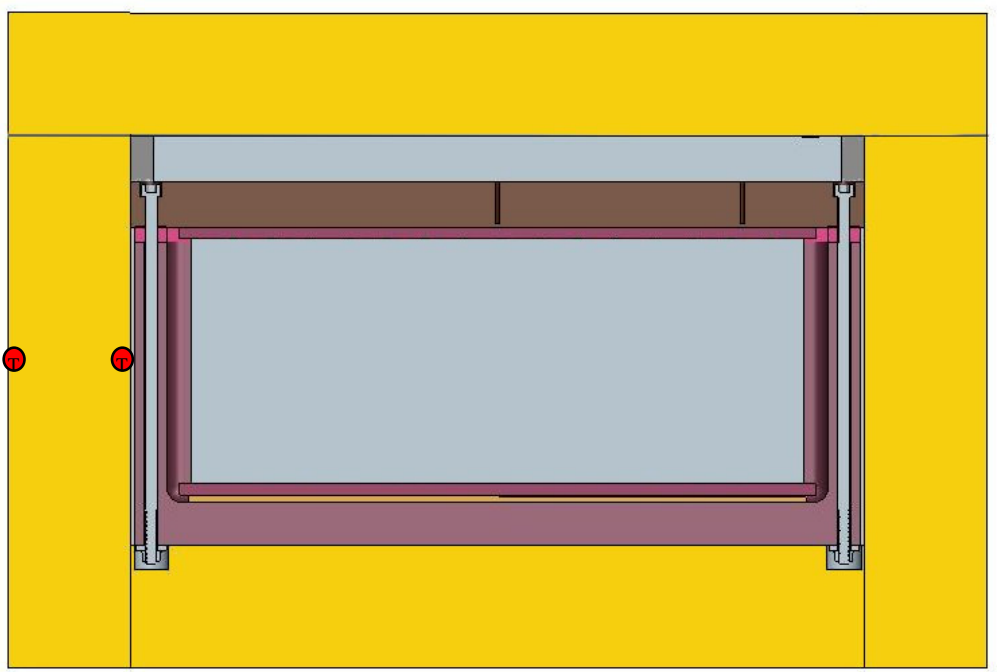

The container is instrumented with 7 type $\mathrm{T}$ thermocouples, one at the bottom center of the container, one approximately corresponding to a 4" distant instrument column, and five equally spaced along one side. Three of these are monitored by the DAQ.

The insulation package has two type T thermocouples arranged as shown in Figure 3 to evaluate heat leak from the test article.

The power to each heater is supplied by a Sorensen XHR 60-18, which is capable of up to $1080 \mathrm{~W}$. The three heaters adhered to each test article heat spreader are connected in parallel to this supply. The Sorensen measures the voltage and current supplied. Sense lines for applied voltage measure the voltage at the heater leads junction to minimize current induced error. The power supply is controlled by, and its measurements are transmitted to the test DAQ.

\section{Chilled Fluid Loop}

The lab fluid loop consists of a main loop with pressure relief, two parallel test loops to which each test article cold plate is attached, and a bypass loop to allow for flow control through the test loops. A chiller supplies cold process fluid to the fluid loop. A schematic of the loop is shown in Figure 4. Temperature, pressure, and mass flow instrumentation is recorded at regular intervals by the DAQ.

The chiller is a two-stage refrigerant, air-cooled, recirculating fluid chiller from SP Scientific, the RC211 ULT Series fitted with a high pressure Tuthill T5.3 constant displacement pump. The process fluid used is Syltherm XLT, a silicone based low temperature heat transfer fluid made by Dow Chemical. The system is designed to condition the process fluid to temperatures between $35^{\circ} \mathrm{C}$ and $-40^{\circ} \mathrm{C}$.

System fluid pressure and temperature are monitored upstream of the bypass at P001 and T01 respectively for software monitoring of process conditions. Temperatures entering and leaving each

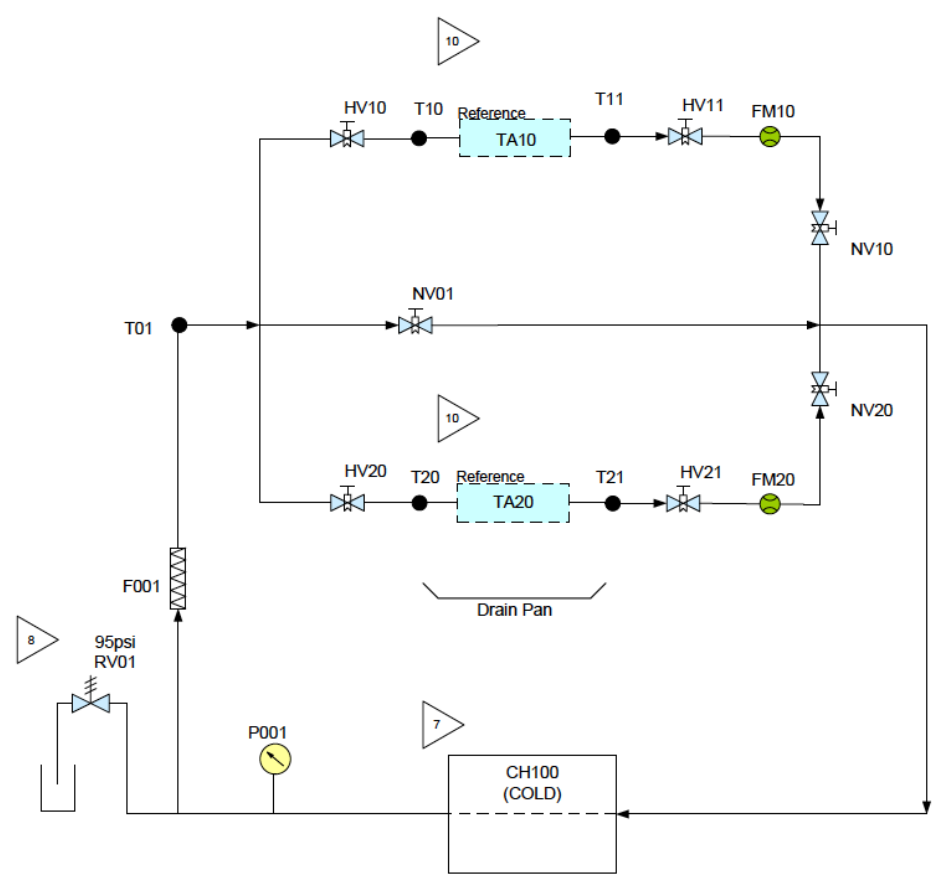

Figure 4. Low Temperature Lab Fluid Loop Test Schematic. 
coldplate, designated TA10 and TA20, are monitored to identify local process temperature and, in conjunction with flow rate measurement at FM10 and FM20, to calculate fluid thermal power removed from the test article. A single needle valve on the bypass loop controls magnitude of flow to the test loops. Needle valves on each test loop allow control of the proportion of flow through the respective test loop. Hand valves located in the test loops allow the coldplates to be isolated for test article removal.

\section{Test Method}

General test operation for freeze/melt cycles, with representative temperatures, is as follows. To begin, the heater is turned on and set to control the test article to an isothermal condition at $35^{\circ} \mathrm{C}$. Next, the heater is turned off and the chiller is turned on. The chiller setpoint is set to $-40^{\circ} \mathrm{C}$, and the flow rate through the test articles is set to $300 \mathrm{lbm} / \mathrm{hr}$ by the position of the valves on the test loop. Once the warmest temperature in the test article falls below $-10^{\circ} \mathrm{C}$ the chiller is turned off. With both the chiller and heater off, the test article is allowed to equilibrate until an isothermal condition is achieved. An isothermal condition is met when all test article temperatures at a point in time have a standard deviation less than or equal to $1^{\circ} \mathrm{C}$. At this time, the heater is turned on at its desired power setting to begin the melt cycle. The heater applies a constant power until the maximum temperature, measured upon the heat spreader, in the test article reaches $35^{\circ} \mathrm{C}$, at which time power level adjusts to maintain this temperature. It continues to control to this temperature until the test article achieves a steady isothermal condition.

In addition to the freeze/melt cycles, two forms of steady state test points are run. First is a steady state in which a steady temperature gradient is established across the test article, a diabatic steady state. These steady state conditions allow an effective thermal conductivity for the water and interstitial material to be determined. The general test operation for the steady state test points is to establish a low temperature at the top of the test article with fluid flowing through the coldplate while establishing a high temperature at the bottom of the test article by controlling the heater to a high temperature setpoint.

The second steady state maintains a constant, isothermal temperature of the test article that is greater than ambient room temperature, an adiabatic steady state. This is performed by applying power to the heaters only to maintain a given temperature. This test allows for the measurement of a thermal resistance to ambient for the insulation package by inferring that the power required to maintain a given temperature is exactly that which is leaking from the system through the insulation package. Figure 5 summarizes typical test conditions.

Each instrument is calibrated prior to testing. Flow meters are calibrated by the JSC building 33 calibration lab. RTD's are calibrated by the JSC Measurements Standards and Calibration Laboratory (MSCL). All thermocouples are checked and all thermocouple channels on the DAQ are calibrated on the bench against an Omega model CL23A digital thermocouple calibrator. The curves produced from each calibration are applied to the DAQ software.

The DAQ software controls the heaters via analog signaling and controls the chiller via RS-232. The test operator inputs heater set temperature, fluid set temperature, heater set power, and while autonomous a

The matrix of test conditions that is applied to each test article is generally as follows: Freeze

- With fluid at $-40^{\circ} \mathrm{C}, 300 \mathrm{lbm} / \mathrm{hr}$

- With fluid at $-35^{\circ} \mathrm{C}, 300 \mathrm{lbm} / \mathrm{hr}$

- With fluid at $-30^{\circ} \mathrm{C}, 300 \mathrm{lbm} / \mathrm{hr}$ Melt

- $\quad 100 \mathrm{~W}$

- $200 \mathrm{~W}$

- $300 \mathrm{~W}$

- $500 \mathrm{~W}$

Diabatic Steady State

(low is fluid, high is heater temperature setpoint)

- $\quad-40^{\circ} \mathrm{C}$ to $-20^{\circ} \mathrm{C}, 400 \mathrm{lbm} / \mathrm{hr}$

- $\quad-25^{\circ} \mathrm{C}$ to $-5^{\circ} \mathrm{C}, 400 \mathrm{lbm} / \mathrm{hr}$

- $\quad-1^{\circ} \mathrm{C}$ to $15^{\circ} \mathrm{C}, 400 \mathrm{lbm} / \mathrm{hr}$

- $\quad-1^{\circ} \mathrm{C}$ to $30^{\circ} \mathrm{C}, 400 \mathrm{lbm} / \mathrm{hr}$

Adiabatic Steady State (test article set temperature)

- $35^{\circ} \mathrm{C}$

- $\quad 40^{\circ} \mathrm{C}$

- $\quad 48^{\circ} \mathrm{C}$

Figure 5. Matrix of Test Conditions.

time based series of these inputs, to control the parameters of the test. The software acquires measurements from all DAQ attached instruments and performs a set of basic calculations, such as applying calibration curves to the raw measurements, determining heater power, and determining fluid power. The resulting data set for two test articles is written to disk in a comma separated format. A second software package written for Octave performs data analysis and reduction procedures including recognizing patterns in the raw data which match each test type, performing test specific calculations, and disseminating the results into concise data sets for further analysis. 


\section{E. Test Matrix}

The matrix of test articles evaluated is shown in Table 1. In this table, $\mathrm{L}$ is the characteristic length for each material. The characteristic length is a characteristic spacing; fin spacing for fins (the inverse of fins per inch, fpi), cell size for honeycomb, and pore size for foams (the inverse of pores per inch, ppi). The metal mass fraction, $\varepsilon_{\mathrm{f}}$, is the percent actual mass of the interstitial material relative to the solid mass of its base metal that would fill the same volume. This is similar to relative density, $\rho_{\text {rel }}$, the ratio of interstitial material density with respect to base metal

Table 1. Test Article Matrix.

\begin{tabular}{|c|c|c|c|c|c|c|c|c|}
\hline $\begin{array}{l}\text { Data } \\
\text { Series }\end{array}$ & Description & Material & $\begin{array}{l}\mathbf{L} \\
\text { (in) }\end{array}$ & $\begin{array}{l}\mathbf{k} \\
(\mathrm{W} / \mathrm{m}-\mathrm{C})\end{array}$ & $\begin{array}{l}\mathrm{c}_{\mathrm{p}} \\
(\mathrm{J} / \mathrm{g}-\mathrm{C})\end{array}$ & $\begin{array}{l}\text { mass } \\
(\mathrm{kg})\end{array}$ & $\begin{array}{l}\mathrm{H}_{2} \mathrm{O} \\
(\mathrm{kg})\end{array}$ & $\varepsilon_{\mathrm{f}}$ \\
\hline Test 1 & Al Fin, 2.5 fpi, 0.040in & Al 1100 & 0.4 & 222 & 0.904 & 1.92 & 5.39 & $10.8 \%$ \\
\hline Test 2 & Al Fin 5 fpi, 0.020in & Al 1100 & 0.2 & 222 & 0.904 & 1.706 & 5.336 & $9.66 \%$ \\
\hline Test 3 & Al Fin 2.5 fpi, 0.020in & Al 1100 & 0.4 & 222 & 0.904 & 0.866 & 5.55 & $4.88 \%$ \\
\hline Test 4 & Al Fin 10 fpi, 0.020in & Al 1100 & 0.1 & 222 & 0.904 & 3.31 & 5.01 & $18.6 \%$ \\
\hline Test 5 & Cu Fin 2.5 fpi, 0.040in & CU11000 & 0.4 & 388 & 0.385 & 6.23 & 5.402 & $10.7 \%$ \\
\hline Test 6 & Al Foam 5 ppi, $10 \%$ & $\mathrm{Al} 6101$ & 0.2 & 218 & 0.895 & 1.734 & 5.766 & $9.80 \%$ \\
\hline Test 7 & Al Fin 5 fpi, 0.040in & Al 1100 & 0.2 & 222 & 0.904 & 3.554 & 4.82 & $20.0 \%$ \\
\hline Test 8 & $\mathrm{Cu}$ Foam 10ppi, 8\% & CU10100 & 0.1 & 387 & 0.385 & 6.896 & 5.27 & $11.8 \%$ \\
\hline Test 9 & $\mathrm{Al} \mathrm{HC} 22.1-1 / 8$ & $\mathrm{Al} 5052$ & 0.125 & 138 & 0.880 & 2.342 & 5.376 & $13.3 \%$ \\
\hline Test 10 & St HC 7.1-3/8 & SS 304 & 0.375 & 16.2 & 0.5 & 0.704 & 5.928 & $1.34 \%$ \\
\hline Test 11 & $\mathrm{Al} \mathrm{HC} \mathrm{1.6-1/4}$ & Al 5052 & 0.25 & 138 & 0.88 & 0.176 & 6.098 & $1.00 \%$ \\
\hline Test 12 & $\mathrm{Al} \mathrm{HC} 7.9-1 / 4$ & Al 5052 & 0.25 & 138 & 0.88 & 0.826 & 5.822 & $4.70 \%$ \\
\hline Test 13 & $\mathrm{Al} \mathrm{HC} \mathrm{8.1-1/8}$ & Al 5052 & 0.125 & 138 & 0.88 & 0.832 & 5.916 & $4.73 \%$ \\
\hline Test 14 & Al Foam 40ppi, 10\% & Al 6101 & 0.025 & 218 & 0.895 & 1.538 & 5.548 & $8.69 \%$ \\
\hline Test 15 & Al Foam 40 ppi, 5\% & Al 6101 & 0.025 & 218 & 0.895 & 0.672 & 5.738 & $3.80 \%$ \\
\hline Test 16 & $\mathrm{Al} \mathrm{HC} \mathrm{22.1-1/8}$ & Al 5052 & 0.125 & 138 & 0.88 & 2.342 & 5.246 & $13.3 \%$ \\
\hline Test 17 & Al Foam 5ppi, 10\% & $\mathrm{Al} 6101$ & 0.2 & 218 & 0.895 & 1.802 & 5.438 & $10.2 \%$ \\
\hline
\end{tabular}

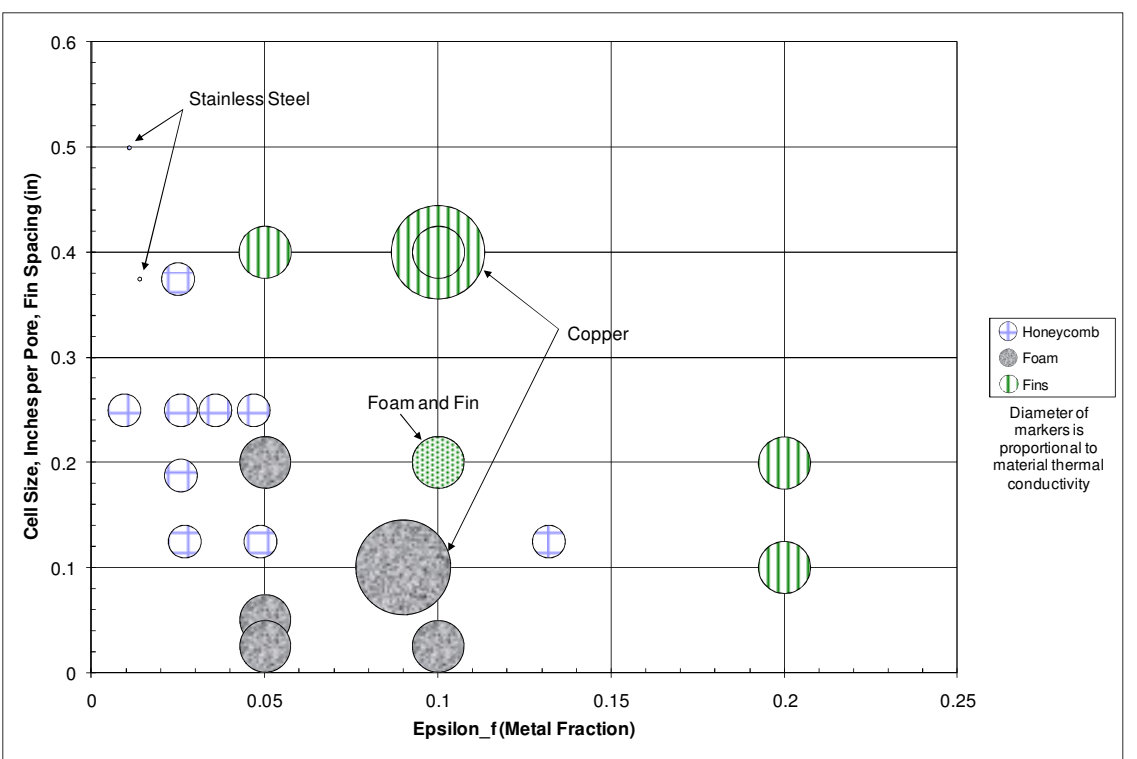

Figure 6. Graphical representation of the test article matrix. density.

The test article matrix is also shown graphically in Figure 6, though not all test articles shown in this figure have been tested. In this figure, each test article is plotted with its metal fraction on the $\mathrm{x}$-axis. Along the $\mathrm{y}$-axis is the characteristic length. The diameter of each marker is proportional to the base material thermal conductivity.

\section{Model Development}

Six test articles with fins are tested, five made from aluminum and one from copper. The fins are either 0.02 " or 0.04 " thick, and are 4" tall. The fin spacing varies from 0.1 " to 0.4 ". The metal fraction varies from 0.05 to 0.2 . Some of the fins have a "lazy ruffled" shape, which means that in the third dimension the fins have a wavy shape, not a planar shape. The pitch (or wavelength) and the amplitude of the waviness is typically 1 " and 0.05 ", respectively. To be modeled exactly, the heat transfer analysis for these fins must be three-dimensional. In our model for the fins, however, we ignore the three-dimensionality of the fins and report only predictions from a 2-D thermal model.

Four test articles with foams are tested, three are aluminum foam and the fourth is a copper foam. The metal fractions are 0.05 and 0.1 . The number of pores per inch varies from 5 to 40 in these foams. We model the foams using the assumption of local thermal equilibrium. In brief, this entails that the energy equation solved is averaged over a spatial volume (called the representative elementary volume) that contains many pores in the foam. Further, local thermal equilibrium assumes that everywhere within the representative elementary volume, the pore level temperature difference between the solid and the fluid is small compared to temperature changes on the scale of the size of the representative elementary volume. Thus the temperature difference between the solid and the fluid is 
ignored and the the porous medium is characterized by a single temperature field that is permitted to vary on the scale of the representative elementary volume. In the test predictions for foams, we have used a $1 \mathrm{~d}$ model where the foam temperature varies only in the vertical direction. The effective thermal conductivity of the foam is a key property in the analysis. We have used the following correlation for Doucel aluminum foams from Schmierer and Razani $^{4}$. In these expressions, $\rho$ is the relative density of the metal in the foam expressed as a percent.

$$
\begin{aligned}
& k_{e}=0.586 \rho+0.8995 \text { (Al fodm in air; } \rho \text { in } \% \text { ) } \\
& k_{e}=0.6356 \rho+1.6742 \text { (Al foam in water; } \rho \text { in } \% \text { ) }
\end{aligned}
$$

The authors obtained these correlations from a linear least squares fit of the data from the literature. In the case of copper foams, we have used the simple expression

$$
k_{e}=C \epsilon_{f} k_{m}
$$

where $\varepsilon_{f}$ is the metal fraction and $k_{m}$ is the native thermal conductivity of the metal. The constant is chosen to be $\mathrm{C}=0.39$. This value of $\mathrm{C}$ is mentioned by Schmierer and Razani to fit well with their measurements (for Al Duocel foam) with a standard deviation of $6 \%$. We assume that there is no difference in the effective thermal conductivity of foam/ice and foam/water regions, though our model can accommodate a difference in these effective conductivities, if they are available.

There are five honeycomb test articles that are tested. four are made from aluminum and one from stainless steel. The cell size (distance between parallel sides) is in the range $1 / 8$ " to $3 / 8 "$, the wall thickness is in the range 0.0007 " to 0.006 ", and the metal fraction is in the range 0.0095 to 0.132 .

Just like the lazy-ruffled fin, a honeycomb structure is inherently a 3-D structure, and requires a $3-\mathrm{D}$ thermal model to be accurately modeled. Honeycombs are typically made from plates or sheets by bending them to form the appropriate shape, and stacked to form a cellular structure. A hexagonal honeycomb together with its unit

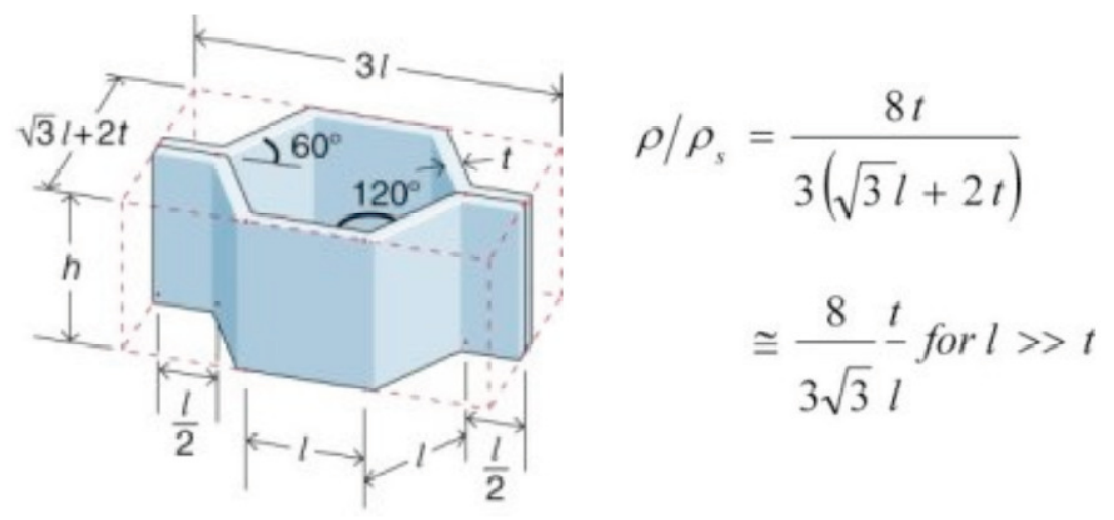

Figure 7. A honeycomb unit cell and an expression for its relative density cell representation is shown in

Figure 7. The expression for the relative density of the honeycomb in terms of geometrical parameters is also shown.

Repeating the unit cell in two dimensions would create the 3-D cellular solid. In this particular hexagonal honeycomb, there is a doubling in wall thickness of every other web which results in an anisotropic character of the material. An alternative manner of depicting the unit cell would be as a regular hexagon, with two parallel walls
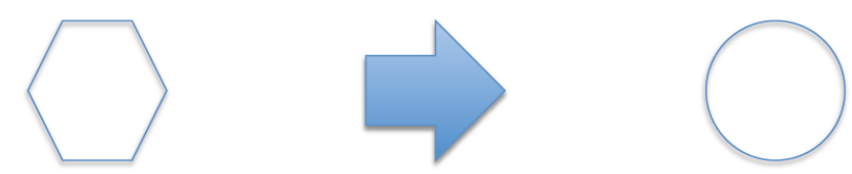
being twice the thickness of the other four walls.

Our predictions, however, are based on a 2-D model for ease of numerical computations. To reduce the honeycomb modeling from 3-D to 2-D, we constructed a cylindrical unit-cell representation of the honeycomb by matching the Figure 8. Simplified representation of the honeycomb PCM volume and metal volume in the unit cell. hexagonal geometry for 2-D modeling

This procedure is illustrated below in Figure 8 and requires matching of the respective crosssectional areas.

By equating the cross-sectional areas of the honeycomb cell (assumed to be a hexagon) and a cylinder, the equivalent radius of the cylinder is obtained as

$$
r_{c}=\left(\frac{\sqrt{3}}{2 \pi}\right)^{0.5} c
$$

where $c$ is the cell size (i.e., the distance between the parallel sides). Similarly, by equating the metal fraction in the two geometries, the cylinder wall thickness can be obtained to be 


$$
t_{c}=\left(\frac{1}{\sqrt{1-\epsilon_{f}}}-1\right) r_{c}
$$

In our model we have assumed that heat is transferred to/from the unit cell across its top and bottom boundaries only. There is thus there no heat flux at the outer wall of the cylinder, i.e., the boundary $r=r_{c}+t_{c}$ is assumed to be adiabatic.

A schematic of the unit cell for fins is shown in Figure 9. The dimensions shown are in meters. Note that the horizontal and vertical length scales differ. What is shown at the left is one half of the fin; the left boundary is the centerline of the fin. Similarly the right boundary is the centerline of the PCM. The fin and PCM are sandwiched between thermal pads at the top and the bottom. The thermal pads ensure that there is good thermal contact with the fin on one side and the heat spreader plates on the other side. The thermal pad may be compressed a little to fit in the space between the fin and the spreader plate. In our model we ignore any local compression of the pads near the fin and assume that its thickness is uniform everywhere.

To solidify PCM that is initially present in the liquid state in the heat exchanger, we assume that the top heat spreader plate is in contact with a cold plate/heat exchanger that is cooled by a chiller. When the PCM is in its solid state, melting is assumed to take place by energizing the bottom heat spreader by a heater.

The centerlines of the fin and PCM are assumed to be adiabatic in our model. Thus the heat transfer to/from the top and bottom boundaries is linked with the internal energy change

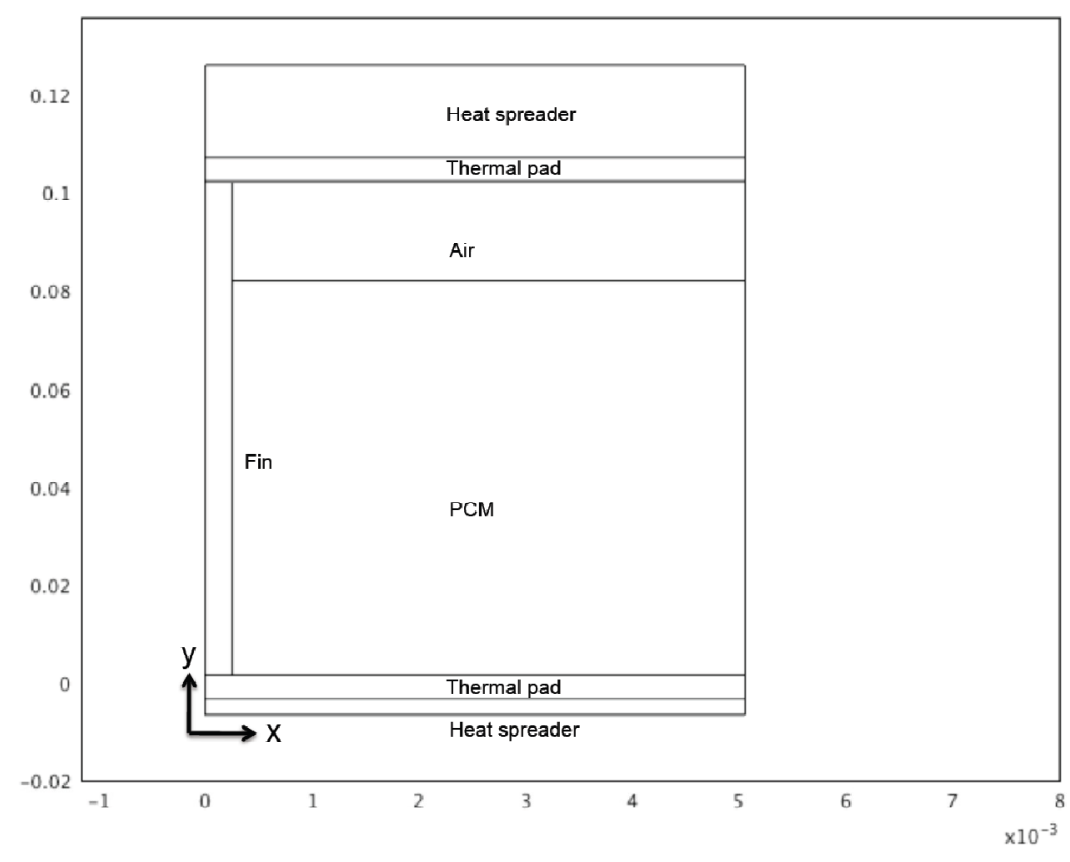

Figure 9. A schematic of the unit cell as modeled for fin geometries. within the unit cell.

\section{A. Approximations in the model}

When heat transfer occurs primarily through the metal, the melt-time can be calculated as follows. The net heat input per unit depth (in the third dimension) to the melting front during a time interval $\Delta t$ is $q W \Delta t$, where $q$ is the heat flux and $W$ is the width. During this time interval the liquid/solid interface in the PCM moves by a distance $\Delta y$. Then energy balance gives

$$
q W \Delta t=(1-\epsilon) W \rho_{p c m} \hat{L} \Delta y
$$

The front propagation velocity can then be expressed as,

$$
V_{\text {front }}=\frac{\Delta y}{\Delta t}=\frac{q}{(1-\epsilon) \rho_{p c m} \hat{T}}
$$

The melt-time, tmelt for a heat sink of height $\mathrm{H}$ is given by

$$
t_{\text {melt }}=\frac{H}{V_{\text {front }}}=\frac{(1-\epsilon) H \rho_{p c m} \hat{L}}{q}
$$

This melt-time can also be considered as the thermodynamic melt-time where there is no resistance to heat transfer and all the heat supplied immediately goes toward phase change. The temperature rise at the heater surface can now be estimated as follows. Assuming all the heat is transferred through the fin only, the heat flux through the fin $q_{f}$ is equal to $q / \varepsilon$. Using quasi-steady conduction yields, 


$$
T_{\text {base }}=T_{m}+\frac{q H}{\epsilon k_{f}}
$$

where $T_{\text {base }}$ is the temperature at the base of the fin, $T_{m}$ is the melting temperature of the PCM, and $k_{f}$ is the thermal conductivity of the fin material.

Consider for solidification the situation where the PCM in liquid state is initially at some ambient temperature and suddenly the top surface temperature is lowered to a temperature $T_{c}$, below the freezing temperature $T_{m}$ of water. The freezing front starts at the top and propagates downward. Assume, for now, that there is no air gap above the PCM. We perform a one-dimensional energy balance as follows. Assume that all the latent heat released at the freeze front is transferred from the freezing front by conduction to the top surface through an already frozen layer. The energy balance can be written as

$$
\left[(1-c) W k_{p c m}+c W k_{f}\right] \frac{\left(T_{m}-T_{c}\right)}{\left(y-y_{0}\right)} \Delta t=(1-c) W \rho_{p c m} \hat{L} \Delta y
$$

The freezing-front propagation velocity is then given by

$$
v_{f f}=\frac{\Delta y}{\Delta \ell}=\left[\frac{\epsilon k_{f}}{1-\epsilon}+k_{p c m}\right] \frac{\left(T_{m}-T_{c}\right)}{\rho_{p c m} \hat{L}\left(y-y_{0}\right)}
$$

This equation can be integrated to show that the freeze-front location as a function of time can be expressed as

$$
y=y_{0}+\sqrt{2 P t}
$$

with

$$
P=\left[\frac{\epsilon k_{f}}{1-\epsilon}+k_{p c m}\right] \frac{\left(T_{m}-T_{c}\right)}{\rho_{p c m} \hat{L}}
$$

The overall freezing time is $t_{\text {freeze }}=H^{2} / 2 P$. Unlike the melting case which applies a uniform heat flux, the freezefront velocity is a function of the front location, and the overall freezing time varies as the square of the heat sink height $H$ compared to the linear variation for melting.

When an air gap is present between the top surface (at temperature $T_{c}$ ) and the surface of the PCM, we need to include the heat transfer resistance due to the air gap in the above analysis. Let $f$ be the fraction of the test article height that is occupied by air (i.e., the air gap is of height $f H$, and the PCM is of height $(1-f) H$ ). The time taken to freeze the PCM is now obtained to be

$$
t_{\text {freeze }}=\frac{\left[\left(f H \frac{\left(\epsilon k_{f}+(1-\epsilon) k_{p c m}\right)}{\left(\epsilon k_{f}+(1-\epsilon) k_{a i r}\right)}+(1-f) H\right)^{2}-\left(f H \frac{\left(\epsilon k_{f}+(1-\epsilon) k_{p c m}\right)}{\left(\epsilon k_{f}+(1-\epsilon) k_{a i r}\right)}\right)^{2}\right]}{\left[\frac{2\left(\epsilon k_{f}+(1-\epsilon) k_{p c m}\right)\left(T_{m}-T_{c}\right)}{(1-\epsilon) \rho_{p c m} \hat{L}}\right]}
$$

\section{B. Boundary conditions}

In modeling the solidification of the PCM, we assume that the test article is initially filled with the PCM in its liquid state to the desired extent (80\% of the height of the test article, with the remaining $20 \%$ at the top occupied by air to allow for expansion of the water once it freezes). The entire contents of the test article (metal, PCM, thermal pads and spreader plates) are assumed to be at a uniform temperature, that is input from the experimental measurements. To initiate freezing, we impose an experimentally measured temperature vs. time profile at the boundary between the top spreader plate and the thermal pad. This corresponds to the center thermocouple on the lid ( $\mathrm{LidC})$ in the experiments. We assume that the temperature of the spreader plate / thermal pad boundary is spatially uniform. There is some variation in the temperature of this thermocouple when compared to that of other thermocouples at other location on the lid, especially early in the cooling process. Nevertheless, we ignore this variation and use the temperature trace from LidC as the boundary condition because the center column thermocouples (TC, C3, C2 C1 and BC) whose traces we compare with are all located directly beneath the LidC thermocouple. In a few cases, the LidC thermocouple traces are not available (test 1, test 4, and test 9). In these cases, we use the information from the other lid thermocouples to impose boundary conditions in the model. In all cases, the bottom boundary of the unit cell (the boundary between the bottom of the delrin box and the surroundings) is assumed to be adiabatic.

Before melting, we assume that the contents of the test article including the PCM are initially at a uniform temperature that is obtained from the experiments. To initiate melting, we assume that a uniform heat flux (the actual value of the heat flux from the heater) is imposed beneath the bottom spreader plate. The bottom boundary of the delrin box and the upper boundary of the top spreader plate at the other end are assumed to be adiabatic. 


\section{Governing equations}

The numerical model is briefly described below for the case of a rectangular fin. The model is a 2-D model. For the cylindrical cell representation of the honeycomb cells we use an axisymmetric model in cylindrical coordinates. In the case of foams, the model is one-dimensional. During melting we assume that a heat flux is supplied at the boundary $y=0$. The remaining boundaries of the unit cell at $y=H_{t o p}$ and $x=0 ; W$ are adiabatic. At the boundary $x=d$ between the metal and the PCM, the temperature and the heat flux are assumed to be continuous. The air gap above the PCM, the thermal pads, and the heat spreader plates are all included in the numerical model. A density change on phase change, however, is not included. In our model heat transfer is solely by conduction - there is no convection of any kind that is considered. Latent heat storage/release is handled by an enthalpy method which incorporates the latent heat as a modification of the specific heat in a small temperature range around the phase change temperature. The energy equation for the fin and the PCM are written below.

$$
\begin{aligned}
& \frac{\partial T_{j}}{\partial t}=\alpha_{j} \nabla^{2} T_{j} \\
& \rho C_{p} \frac{\partial T}{\partial t}=\nabla \cdot(k \nabla T)-\hat{\rho} \hat{L} \frac{\partial \gamma}{\partial t}
\end{aligned}
$$

$T_{f}$ and $T$ refer to the fin and PCM temperature, respectively. $\gamma$ is the liquid fraction and is equal to 1 when the PCM is a liquid and 0 when it is a solid. It can be written as $\gamma=U\left[T-T_{m}\right]$ where $U[x]$ is the Heaviside unit step function, and $T_{m}$ is the melting temperature. For numerical purposes we write $\gamma=1 / 2\{1+\tanh [a(T$ $\left.T_{m}\right)$ ]\}, where $a$ is a constant chosen to be appropriately large. $\hat{L}$ is the latent heat for phase change. $\hat{\rho}=\rho_{l}$ for melting (and $\rho_{s}$ for solidification; we have assumed $\rho_{l}=\rho_{s}$ ). We have used $a=50$. The resulting liquid fraction is plotted in Figure 10. We see that the phase change occurs within approximately $\pm 0.1^{\circ} \mathrm{C}$ from $T_{m}$.

The working form of the energy equation in the PCM is written below. A

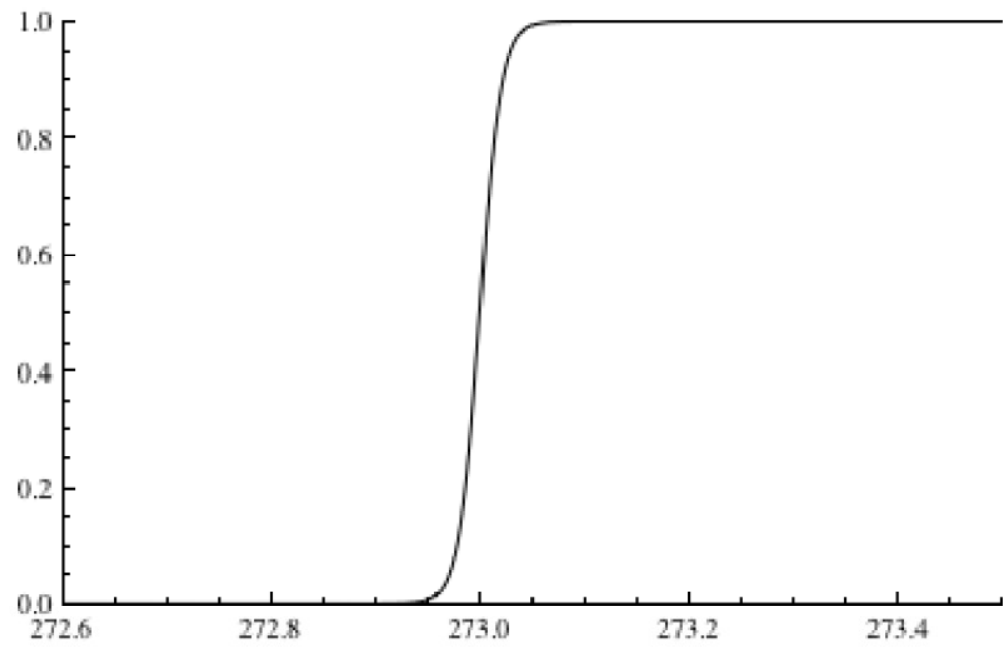

Figure 10. Liquid fraction by temperature change in the heat capacity and thermal conductivity between the solid and liquid is accommodated. This is accomplished by representing these quantities as being dependent on the liquid fraction $\gamma$.

$$
\begin{gathered}
\left\{\rho_{s} C_{p_{s}}\left[1+(\lambda-1)\left\{\frac{1}{2}+\frac{1}{2} \tanh \left[a\left(T-T_{m}\right)\right]\right\}\right]+\frac{a}{2} \hat{\rho} \hat{L} \operatorname{sech}^{2}\left[a\left(T-T_{m}\right)\right]\right\} \frac{\partial T}{\partial t}- \\
k_{s} \frac{\partial}{\partial x}\left[\left(1+(\beta-1)\left\{\frac{1}{2}+\frac{1}{2} \tanh \left[a\left(T-T_{m}\right)\right]\right\}\right) \frac{\partial T}{\partial x}\right\rfloor+k_{s} \frac{\partial}{\partial y}\left\lfloor\left(1+(\beta-1)\left\{\frac{1}{2}+\frac{1}{2} \tanh \left[a\left(T-T_{m}\right)\right]\right\}\right) \frac{\partial T}{\partial y}\right\rfloor
\end{gathered}
$$

where $\lambda=\frac{\rho_{l} C_{p l}}{\rho_{s} C_{p s}}$ is the ratio of liquid to solid PCM thermal capacity, and $\beta=\frac{k_{l}}{k_{s}}$ is the ratio of liquid to solid PCM thermal conductivity. The initial and boundary conditions are

At $\mathrm{t}=0, T_{f}=T=T_{0}$

At $\mathrm{y}=0,-k_{f} \frac{\partial T_{f}}{\partial y}=-k \frac{\partial T}{\partial y}=q$

$\Lambda \mathrm{t} \mathrm{y}=\mathrm{H}_{\text {top }}$, heat flux $=0$

At all internal boundaries, we have imposed continuity of temperature and the heat flux. In the case of freezing, the formulation is the same except that the boundary conditions are modified to

At $y=\mathrm{II}_{\text {top }}, T=T_{w}$

At $\mathrm{y}=0$, heat flux $=0$

The system of equations is solved numerically using COMSOL. We have not systematically checked the grid dependence of the calculations. In one simulation for melting with aluminum fins, a superficial comparison of 


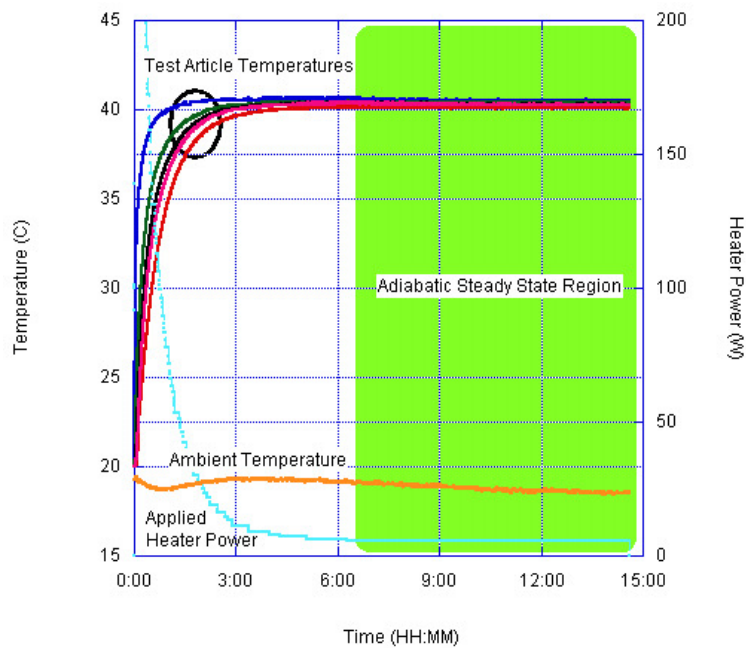

Figure 11. A representative Adiabatic Steady State data set ambient. The heater power added approaches the system heat leak as the test article temperature approaches the set temperature. Figure 11 shows a representative data set from the testing to better illustrate the test.

The adiabatic region is determined programmatically by the Octave data reduction software. The software finds regions of heater-only operation in the data sets that meet a zero-slope criteria with temperatures greater than ambient over at least a $2 \mathrm{hr}$ time period. The final $40 \%$ of a data set matching these criteria becomes the steady state region for calculations.

A thermal resistance is determined as

$$
R_{m}=\frac{T_{a m b}-T_{m}}{Q_{\text {heater }}},
$$

where, $T_{a m b}$ represents the mean ambient temperature, $T_{m}$ the mean test article temperature, and $Q_{\text {heater }}$ the mean heat applied by the heater to the test article over the test period. The results of this test are shown in Figure 12. The uncertainty of these measurements is influenced by the heater control algorithm and a relatively small magnitude of heat leak.

The results suggest a consistent heat leak through all testing within the bounds of uncertainty. An average of the thermal resistance measurements,

$$
R_{m}=4.278 \frac{C}{W} \pm 0.1277 \frac{C}{W},
$$

is applied in subsequent calculations to account for heat leak to the test article from ambient. Uncertainties are determined on a $95 \%$ confidence interval, i.e. on a $2 \sigma$ interval.

\section{B. Diabatic Steady State}

The purpose of the diabatic steady state test is two-fold. This test applies heat via the heater and removes heat via the cold plate allowing an energy balance consideration of the test article as well as a measurement of the composite conductivity of the test article. Figure 13 offers a representative diabatic steady state test data set. Heater and fluid applied power approach a constant value as test article temperatures approach steady state. Ideally, where there is no heat leak or bias, the fluid and heater power approach exactly the same value; e.g. all heat added to the test article by 
the heater would exactly be removed by the cold plate. In fact, however, the cold plate removes more heat due to

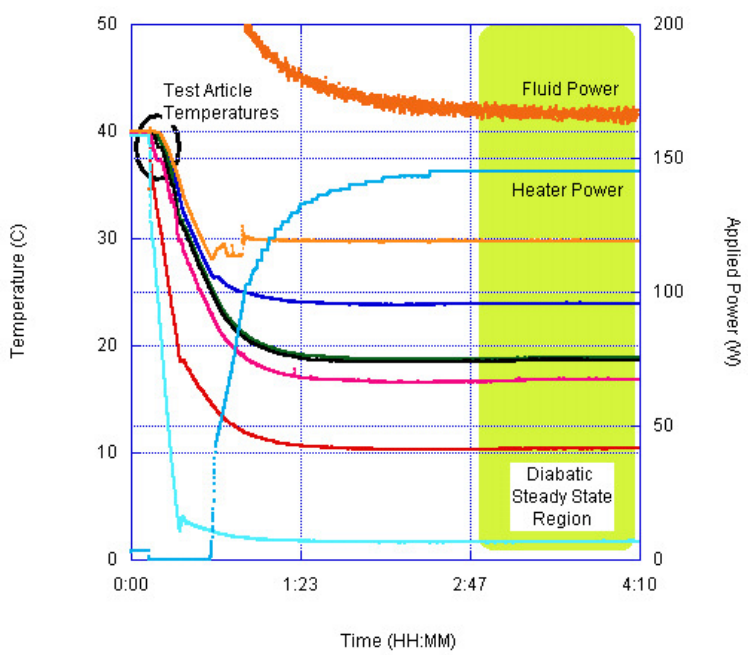
real heat leaks into the control volume.

When considering an energy balance of this system, imagine a volume that contains the test article and includes the chiller inlet and outlet tube lengths extending 4 to 8 inches upstream and downstream of the test article to the location of the fluid RTDs. Including these inlet and outlet lengths is significant as, in addition to the heat leak identified from the test article to the environment, there is a heat leak between the cold fluid, when the fluid loop is active, and ambient along the upstream tube length from the RTD to the insulation package and again along the downstream tube length from the insulation package to the

Figure 13 A Typical Steady State Diabatic data set

downstream RTD. A heat balance calculation of the form

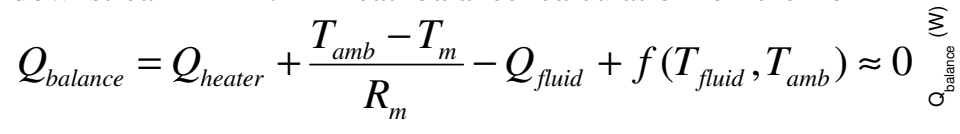

where $Q_{\text {fluid }}$ is the heat removed by the chilled fluid loop, when considered with respect to the temperature difference between the chilled loop fluid and ambient, $\Delta T_{\text {fluid_leak }}=\left(T_{\text {amb }}-T_{\text {fluid }}\right)$,

where $T_{\text {fluid }}$ is the inlet fluid temperature to the cold plate, shows significant correlation as seen in Figure 14. This correlation indicates that there is both a test location specific temperature-dependent heat leak from the environment to the chilled fluid loop, represented in the slope of the fit, and that there is a measurement bias in the fluid loop, represented in the y-offset of the fit. Linear fit coefficient values and their

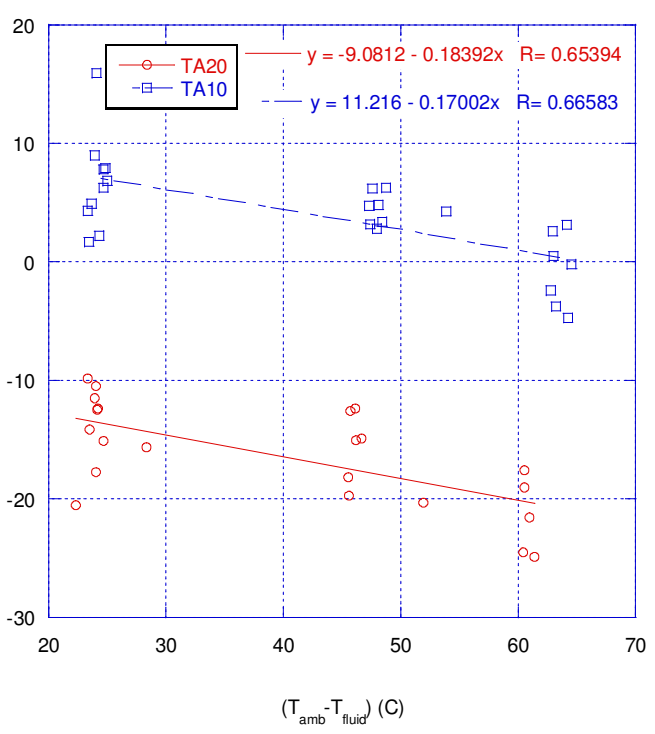

Figure 14 A significant correlation found between the heat balance and fluid temperature difference with respect to ambient temperature.

corresponding error is provided in Table 2. Applying these to correct measurements taken while the fluid loop is active corrects the heat balance for the system so that it approaches zero as prescribed by the first law. In other words, the complete heat balance, accounting for all heat paths entering and exiting the test article control volume, includes a function of the form

$$
f\left(T_{\text {fluid }}, T_{\text {amb }}\right)=\left(m \cdot \Delta T_{\text {fluid_leak }}+b\right),
$$

where $\mathrm{m}$ and $\mathrm{b}$ are the fit values as defined in Table 2 .

Table 2 Fluid loop heat leak fit and error per test article. Location refers to Figure 4.

\begin{tabular}{|c|c|c|c|c|}
\hline & \multicolumn{2}{|c|}{ Location TA10 } & \multicolumn{2}{|c|}{ Location TA20 } \\
\hline$y=m x+b$ & $\mathbf{m}(\mathrm{W} / \mathrm{C})$ & b $(\mathbf{W})$ & $\mathrm{m}(\mathrm{W} / \mathrm{C})$ & $\mathbf{b}(\mathbf{W})$ \\
\hline Linear Fit & $\begin{array}{l}-0.1700 \\
\end{array}$ & 11.23 & -0.1839 & -9.081 \\
\hline Error & \pm 0.03973 & \pm 1.832 & \pm 0.04758 & \pm 2.023 \\
\hline
\end{tabular}

The composite conductivity of the PCM and interstitial material is determined as

$$
K_{\text {comp }}=\frac{k_{\text {comp }} \cdot A}{H}=\frac{q_{\text {heater }}}{\left(T_{\text {mean }, \text { btm }}-T_{\text {mean }, \text { top }}\right)}
$$

where $A$ is the heat spreader contact area, $H$ is the height of the interstitial material, and the respective temperatures are measured on the interstitial material. The value of $K_{\text {comp }}$ can be predicted via the electrical analogy as a set of parallel and series resistances. The diagram in Figure 15 shows the components of the resistance network considered in this prediction; these include the PCM water and interstitial material, the surrounding liquid water, and the delrin container material.

12

American Institute of Aeronautics and Astronautics 


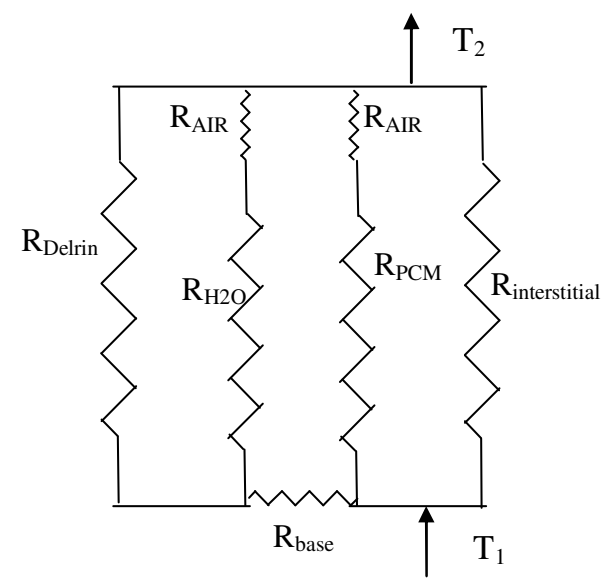

q

Figure 15 An illustration of the set of thermal resistances used to predict the composite conductivity of the test articles.

temperature of $10^{\circ} \mathrm{C}$ shows that the error associated with the effective conductivity (as compared to the prediction) increases with increased fin spacing. The effective conductivity for a fin spacing of $10 \mathrm{fpi}$ is close to the predicted conductivity. However, the error associated with a fin spacing of $2.5 \mathrm{fpi}$ is much greater than both $10 \mathrm{fpi}$ and 5 fpi. These differences in the liquid phase indicate that heat transfer (or effective conductivity) is enhanced by natural convection as one would expect a larger convective heat transfer coefficient for wider convection cells.

One of the objectives of this testing is to investigate tradeoffs among different types of interstitial materials, such as fins, foam, and honeycomb. Figure 17 shows a plot of the experimental composite conductivity normalized by the conductivity of the interstitial material versus the relative density (mass fraction) of the interstitial material. Only values measured in solid ice are included so that convection effects are not considered. Aluminum is indicated by blue symbols and copper is indicated by brown symbols. Fins are indicated by squares, foam is indicated by open circles, and honeycomb is indicated by diamonds. For fins and

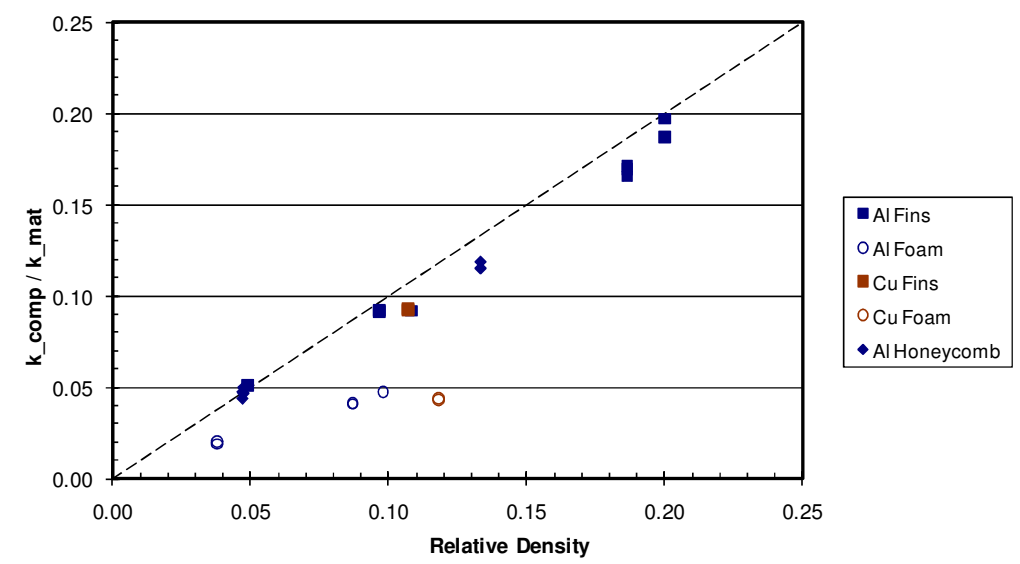

Figure 17. Experimental composite conductivity (solid ice only) for various interstitial materials and configurations.

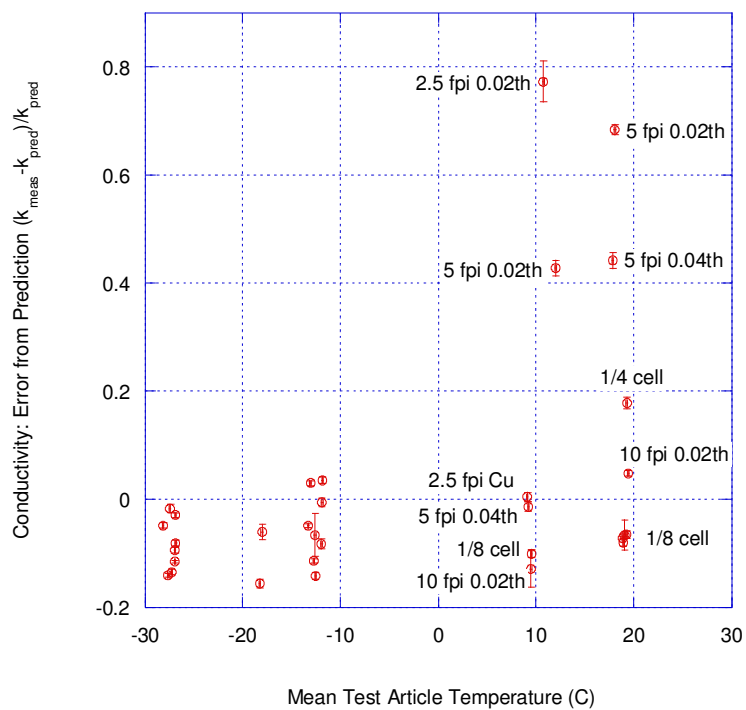

Figure 16. Difference on the Predicted Composite Conductivity for Fins and Honeycomb conductivities fall near the dotted diagonal line indicating that a one-dimensional approximation for the composite conductivity is fairly accurate. However, composite conductivities for the metal foams are lower. It might be noted that this experiment is designed to judge behavior of these materials in an approximately 1-D thermal field, and that the advantages of the 3-D structure of foams will not be made apparent from the results of this experiment.

Since the purpose of adding interstitial materials to a PCM is to increase the composite conductivity in the direction of heat transfer, this shows that for a given mass fraction of interstitial material, fins and honeycomb are more effective at increasing the composite conductivity in the design envisioned here than is foam. 
In addition to tradeoffs among different types of interstitial materials, another test objective is to investigate tradeoffs between interstitial material thickness and spacing. Figure 18 shows a plot of the same data from Figure 17 except only for the aluminum fins. Here in this plot, fins with a fin thickness of 0.020 in are indicated by blue diamonds, while fins with a thickness of 0.040 in are indicated by pink squares. No obvious differences between these two fin spacings are evident.

\section{Thaw Transients}

The thaw transient test applies a constant heat flux boundary condition to the lower surface of the interstitial material. This test affords a good measure of energy capacity in the test article, and a very comfortable data set for correlation to the conduction based model developed here as it initiates in the ice phase, which is conduction dominated. An example thaw data set is shown in Figure 19

An exercise to verify the integrity of the data which compares an experimentally determined heat of fusion for water with the accepted value is performed on this data set. The results are shown in Figure 20. The good correlation of these results, typically inside of $5 \%$ difference, reflects the high certainty and well defined control volume about the heater. Note the data for test number 6 , an aluminum foam which was found to have penetrated the upper TIM during testing and thus has no contact with the heater TIM. The data for this test shows an under prediction of the heat of fusion by approximately $10 \%$. It is believed that this might be explained by a higher heater temperature at a given heat rate due to the increased thermal resistance of the test article which, as described above, results from a gap between the lower TIM and the interstitial material. A higher heater temperature may reduce the heat leak, so it might be expected that the heat leak correlation overcorrects the measurement. Unfortunately, the same behavior does not hold for test 10 or 11 , which also penetrate the upper TIM, so it is not clear that this is the most accurate explanation.

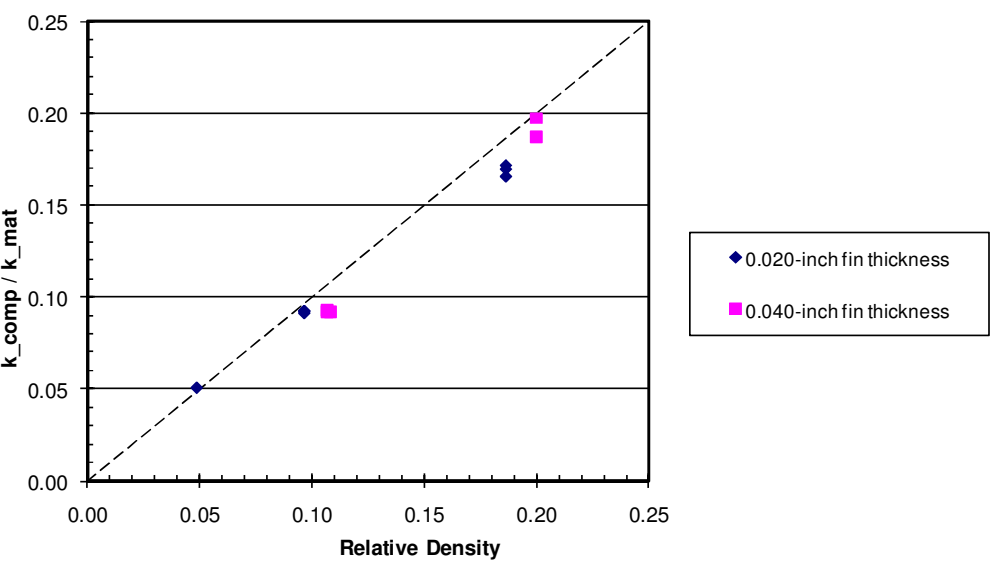

Figure 18. Experimental composite conductivity (solid ice only) for luminum fins.

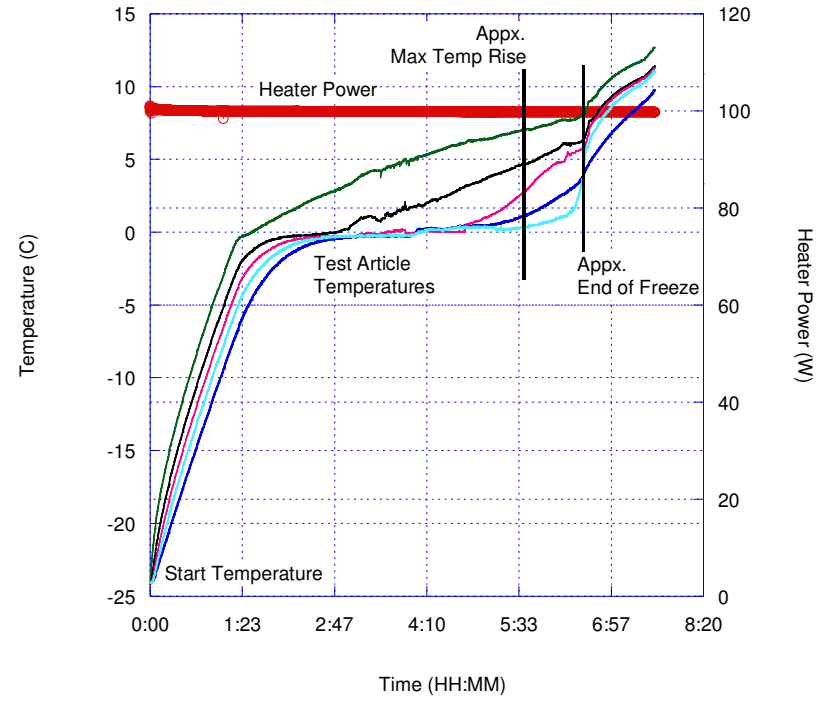

Figure 19 An example thaw data set 
A key criteria in PCM design is the temperature rise across the heat exchanger for a given heat flux. For each thaw transient the data reduction software identifies the greatest temperature rise across the interstitial material. Figure 22 offers a conduction derived correlation for the maximum temperature rise among all materials. The abscissa reflects the balance of Fourier's law of conduction manipulated to solve for temperature rise where the composite material conductivity is represented by $k_{\text {comp }}=k_{e}=C \cdot \varepsilon_{f} \cdot k_{m}$,

where $C$ reflects the mass fraction of material which conducts heat along the direction of heat transfer. In fins and honeycomb, where all or most material is aligned in the direction of conduction, the term $\mathrm{C}$ is unity. In foams, this term is taken here to be 0.33 as recommended by the material manufacturer, with the rationale that in the 3dimentional foam lattice approximately $1 / 3$ of the material is oriented along a given direction of heat transfer.

The correlation arrive at in Figure 22 is simple, bringing together all materials with minimal geometry specific parameters. The fit would be expected to follow a straight line where dominated by conduction, though, as observed before, natural convection influences the system where liquid is present. The greatest temperature rise comes toward the end of the thaw transient where much or most of the ice has thawed. In a conduction dominated system the maximum temperature would be expected at the point where all PCM material has thawed since the conductivity of water is substantially less than that of ice. In these tests the point in time of maximum temperature rise may come earlier, where ice is still present, since natural convection enhances the transfer of heat in the liquid region and buoyancy can detach ice from the interstitial material. Natural convection correlations generally conform to a power law; this data finds good agreement to a power fit.

There are indications in the data that the above correlation does not entirely capture all dependencies. In particular, with natural convection, correlations for the Nusselt number in rectangular cavities include a dependence on the aspect ratio of the cavity ${ }^{5}$. With the test articles having a fixed height, the aspect ratio is determined by a characteristic length such as the fin spacing, suggesting that such a characteristic length carries considerable weight

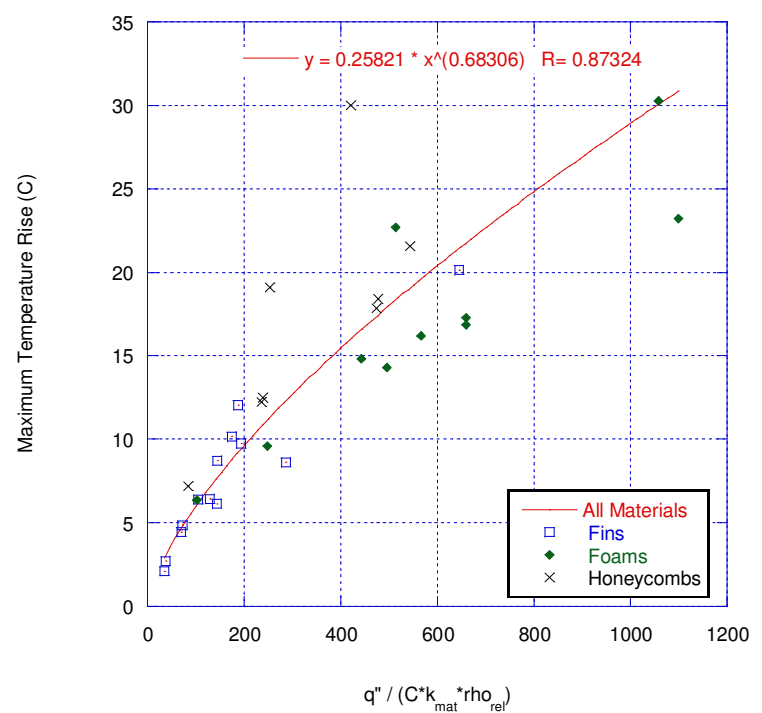

Figure 22 Correlation for temperature rise versus conduction figure of merit.

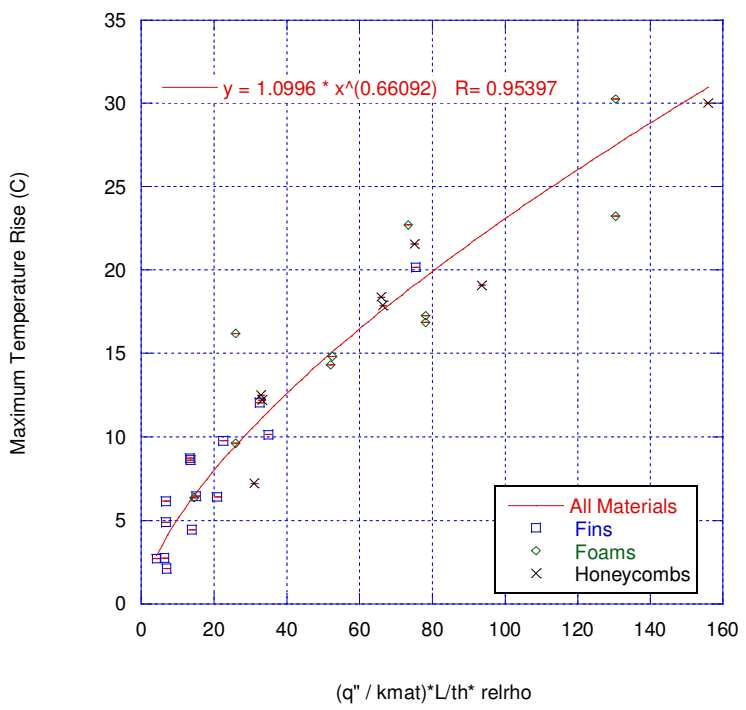

Figure 21 Correlation for temperature rise versus geometric figure of merit 
in enhancing the heat transfer in a system. Honeycomb materials of $1 / 8$ in cell size appear to quell natural convection, as observed in Figure 16, and these data follow a nearly straight line in Figure 22. Figure 21 suggests a correlation with respect to a geometric dependent figure of merit that shows a better qualitative fit. This figure of merit introduces a characteristic length, $L$, which describes fin spacing, cell size, or pore size for each respective interstitial type. A characteristic thickness term, th, describes fin thickness, wall thickness, or estimated lattice element thickness for each interstitial type.

\section{Model Prediction of Thaw Transients}

The general correlation of the model predictions to experimental data is described here. Figure 23 provides an example of the behavior of the model relative to the experiment.

\section{Pre-melting behavior}

In all tested cases the agreement in the pre-melting temperature traces between the predictions and the experiments is very good. This indicates that the thermal masses of the materials within the test box that absorb the heat from the heater

are modeled correctly. We found that the delrin box itself absorbs a significant amount of the heat from the heater.

2. Behavior during phase change

In almost all the cases, there is very good agreement between predictions and measurements for the time at which melting starts (see Table 2). This is the time at which the thermocouple $\mathrm{BC}$ first reaches a temperature of $0^{\circ} \mathrm{C}$. One exception is Test 6 - though the difference between the observed and

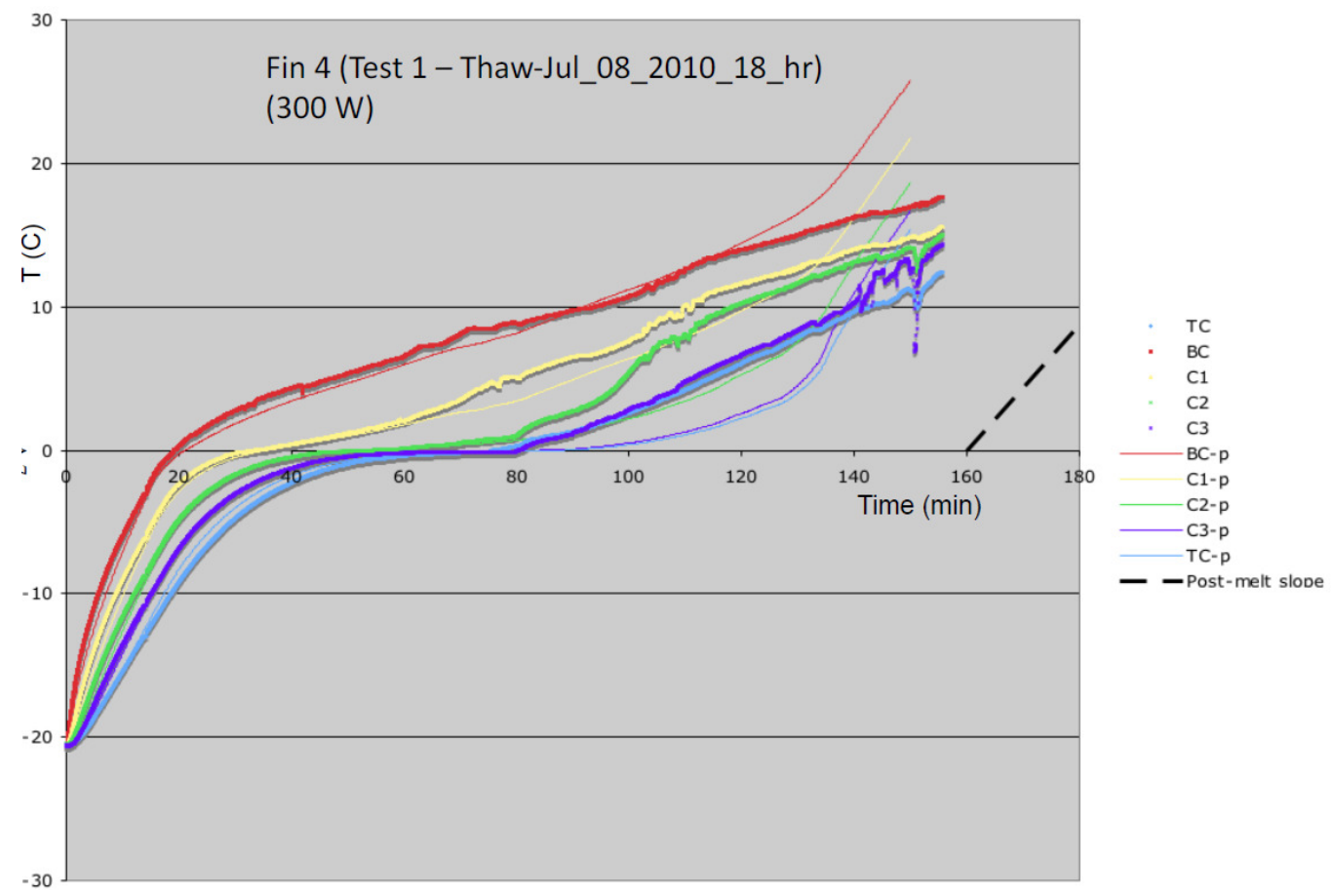

Figure 23. An example comparison of experimental data and model prediction.

predicted temperature at the $\mathrm{BC}$ thermocouple location is less than $2^{\circ} \mathrm{C}$, the measured $\mathrm{BC}$ temperature hovers slightly below $0^{\circ} \mathrm{C}$ for an extended period, and the difference between the start of the melting times is around 36 min.

Table 2 also shows the comparison between predictions and measurements for the time for completion of melting. We define this to be the time when the slopes of the temperature traces change rapidly after thermocouple TC reaches a temperature of $0^{\circ} \mathrm{C}$. The thermodynamic estimate of the melting time, which is the time needed to supply enough heat from the heater necessary for the latent heat of phase change, is also displayed in Table 2. Except for test 1 and test 5, the predicted and observed duration of melting are in reasonable agreement. For test 1 and test 5 the observed melting duration is significantly less than the thermodynamic estimate. This is unlikely, suggesting that this estimate is incorrect (the thermodynamic estimate for Fin 4 and Fin 6 is based on a PCM height of 3.2", fin volume fraction of 0.1 , and a heater power input of $300 \mathrm{~W}$ that is spread over a 10 " by 10 " heater area). In the other cases, the predicted/observed melting duration is larger than the thermodynamic estimate because additional heat from the heater is required for sensible heating of the contents of the test box.

During phase change, the predicted temperature traces as well their slopes compare well with the experimental results. The maximum deviation is about $5^{\circ} \mathrm{C}$, and the typical deviation is less than $2-3^{\circ} \mathrm{C}$. In a few cases (late stages of melting in test 15; a good portion of the melting duration for test 11) the deviation between the predicted and measured temperature is unduly large, especially for the $\mathrm{BC}$ and $\mathrm{C} 1$ temperature traces. For test 11, there is good 
Table 3. Comparison of melting times

\begin{tabular}{|c|c|c|c|c|c|c|c|}
\hline \multirow[b]{2}{*}{ Test article } & \multicolumn{2}{|c|}{ Melting time start ( $\mathrm{min}$ ) } & \multicolumn{2}{|c|}{ Melting time end ( $\mathrm{min}$ ) } & \multicolumn{2}{|c|}{ Melting duration (min) } & \multirow{2}{*}{$\begin{array}{c}\text { Thermodynamic } \\
\text { melting duration } \\
\text { (min) }\end{array}$} \\
\hline & Prediction & Experiment & Prediction & Experiment & Prediction & Experiment & \\
\hline Fin 1 & 87 & 92 & 412 & 393 & 325 & 301 & 277 \\
\hline Fin 2 & 20 & 17 & 177 & 145 & 157 & 128 & 131 \\
\hline Fin 3 & 21 & 19 & 81 & 78 & 60 & 59 & 47 \\
\hline Fin 4 & 21 & 19 & 134 & 81 & 113 & 62 & 88 \\
\hline Fin 5 & 16 & 15 & 77 & 67 & 61 & 52 & 47 \\
\hline Fin 6 & 35 & 31 & 142 & 107 & 107 & 76 & 88 \\
\hline Foam 3 & 29 & 36 & 390 & 394 & 361 & 358 & 277 \\
\hline Foam 4 & 71 & 107 & 361 & 376 & 290 & 269 & 263 \\
\hline Foam 6 & 9 & $* *$ & 120 & 119 & 111 & $* *$ & 88 \\
\hline HC 5 & 73 & 88 & 517 & 482 & 444 & 394 & 289 \\
\hline $\mathrm{HC} 9$ & 26 & 23 & 150 & 135 & 124 & 112 & 84 \\
\hline
\end{tabular}

agreement in the $\mathrm{BC}$ temperature trace until $t=200 \mathrm{~min}$ and in the $\mathrm{C} 1$ trace until $t=300 \mathrm{~min}$. After these times, the predicted temperatures continue to rise, but the measured temperatures level off. This behavior is likely due to the penetration of the upper TIM by the interstitial material which left a gap between the material and the lower TIM allowing cold liquid into the vicinity of the heater.

\section{Post-melting behavior}

The agreement in the post-melting behavior between the predictions and the experiments is not ideal. The slope of the predicted temperature profile is sharper than what is measured in all cases. In order to determine whether the sides of the delrin box and the amount of water between the test article and the delrin box sides (i.e., outside the test article but within the box) plays a role in the discrepancy, we performed a theoretical analysis of the post-melt temperature slope including the thermal mass of the delrin box sides and the excess water. To eliminate ambiguity, we used the measured mass of the test article, cold plate, heat spreader plates, thermal pads, delrin box, and the amount of water used in this analysis. It may be shown that when there is no heat loss to the surroundings the eventual (i.e., steady-state) post-melt temperature variation can be represented as $T(x, t)=\frac{\dot{Q}}{\Sigma m c_{p}}+\theta(x)$, where $\dot{Q}$ is the heater power, $\sum m C_{p}$ is the total thermal mass of the contents of the box, and $x$ represents spatial location within the test box. Curiously, such a relationship may also be expected to hold even if there is convection within the PCM. Thus the slope of the post-melt temperature traces depends only on the heater power and the total thermal mass. The post-melt slope thus determined is shown towards the right end of the chart in Figure 23 to avoid clutter. Note that the actual temperature level depends on $\theta(x)$ which we have not determined. Thus, other than the slope, the placement of this curve on the charts is arbitrary. The post-melt slope is close to but slightly smaller than what is predicted numerically. This is because the thermal mass of the sides of the delrin box and the excess water mentioned above are not included in the numerical computations. The estimated post-melt slope is typically sharper than what is observed from the experimental thermocouple traces.

\section{E. Design Space Considerations}

A useful design space can be presented using the experimental correlations arrived at for the parametric dependencies of the interstitial material. A recommended approach would be to first consider the simpler conductive figure of merit to narrow design considerations. Figure 25 and Figure 24 provide a useful space for considering the relative merit of material selection. 
Typical requirements for a PCM heat exchanger specify a maximum temperature rise at a given heat flux, represented in the abscissa of Figure 25. The engineer is tasked with selecting a material that affords a high specific energy content to arrive at a design having the least mass required to meet an energy storage requirement. The specific latent energy, represented in the ordinate of both figures, describes the latent energy of fusion contained by the water with respect to the total mass of both water and interstitial material. Given these figures, under most conditions, aluminum has a considerable specific energy advantage. Copper only becomes a reasonable choice at very low relative densities, below $\sim 1 \%$. With a material selected, and from Figure 24 a relative density targeted, the engineer may then address the relative merits of geometry via the geometric figure of merit from Figure 21. It is important to remember that the design space represented by these figures applies only to a 1-g environment and that the model predictions only apply to a $0-\mathrm{g}$ environment or a design which quells natural convection.

\section{Conclusions}

This document describes a desktop experiment which measured the performance of a water Phase Change Material (PCM) heat exchanger with many different interstitial materials and interstitial geometries. The experiment provides data for the correlation of a modeling effort which seeks to predict performance of the same system from first principles. The objective of both efforts is the development of a set of tools for design evaluation and prediction in order to guide the development of a water PCM heat exchanger for future exploration vehicles.

The experiment defines a control volume and through correlations developed from the various tests performed closes the first law heat balance of this volume for a well defined system. This is verified by a measured heat of fusion value for water from thaw tests that is within $\pm 5 \%$ of the accepted value. Testing measures composite conductivity, energy storage, and temperature rise in the test articles. Measured conductivities of solid ice PCM generally agree with first principles predictions within $10 \%$ for a conduction dominated system. The model developed here has been shown to predict thaw transients well under most circumstances, though differences arise in the liquid phase where natural convection evident in the experiment is not modeled. The model developed and correlated here will guide future design efforts. Experimental correlations are applied to create a useful design space to identify key parameters for heat exchanger design based on expected design requirements.

\section{References}

\footnotetext{
${ }^{1}$ Lee, S., "REDU Thermal-Vacuum Test Report" CTSD-ADV-742. September 15, 2009.

${ }^{2}$ Lee, S. A., Leimkuehler, T.O., Stephan, R., Le, H. V., "Thermal Vacuum Test of Ice as a Phase Change Material Integrated with a Radiator" AIAA-2010-6113. 40th International Conference on Environmental Systems (ICES), Barcelona, Spain, July 2010 .
} 
${ }^{3}$ Bledjian, L., Hale, D. V., Hoover, M. J., O’Neill, M. J., "Phase-Change Materials” Spacecraft Thermal Control Handbook edited by David G. Gilmore, Vol. 1, $2^{\text {nd }}$ ed., 2002, Chapter 11.

${ }^{4}$ Schmierer, E.N., Razani, A., "Self-Consistent Open-Celled Metal Foam Model for Thermal Applications" ASME JHT 128 , 1194-1203, 2006.

${ }^{5}$ Incropera, F. P., DeWitt, D. P., Bergman, T. L., Lavine, A. S., Introduction to Heat Transfer, $5^{\text {th }}$ ed., Wiley and Sons, New York, 2007. 